\title{
OBSERVATIONS AND ANALYSIS OF TURBULENT WAKE OF WIND TURBINE BY COHERENT DOPPLER LIDAR
}

\author{
Songhua $\mathrm{Wu}^{1 *}$, Jiaping Yin ${ }^{1}$, Rongzhong $\mathrm{Li}^{2}$, Xitao Wang ${ }^{2}$, Bingyi Liu ${ }^{1}$, Jintao Liu ${ }^{1}$ \\ 1. Ocean Remote Sensing Inst., Ocean University of China, Qingdao 266100, China, \\ 2. Seaglet Environmental Technology Inc. Qingdao 266100, China, \\ *Email:wush@ouc.edu.cn
}

\begin{abstract}
Turbulent wake of wind turbine will reduce the power output of wind farm. The access to real turbulent wake of wind turbine blades with different spatial and temporal scales is provided by the pulsed Coherent Doppler Lidar (CDL) which operates by transmitting a laser beam and detecting the radiation backscattered by atmospheric aerosol particles. In this paper, the authors discuss the possibility of using lidar measurements to characterize the complicated wind field, specifically wind velocity deficit by the turbine wake.
\end{abstract}

\section{INTRODUCTION}

Concentration of global energy shortage is closely linked with our living and has become a concern to scientists because of the "Greenhouse Effect". Wind energy industry is growing fast as a sustainable energy source. Because of the obstacle of turbine blades, when the turbine extracts power from the wind, a turbulent wake evolves downwind of the turbine. The power output of the whole wind farm operating within the turbulent wake will be decreased comparing to the turbine operating in the free wind. According to the study by Barthelmie et al., with spacing between the turbines of 4-8 D (Diameter of turbine scanning plane), power dissipation due to wind turbine wakes can be $5 \%-15 \%$. Spacing of turbines beyond $8-10 \mathrm{D}$ is unlikely due to the high cost of installing cables [1]. Furthermore, the wake effect can lead increase of turbulence induced stress and reduce the life time of wind turbines and their components in wind farm. Studies on the turbulent wake are significant for optimizing the turbine arrangement and power output in a wind farm [2].

Turbulent wake characteristics of wind turbines have been studied since 1980s. Current industry standard for wind turbine wake measurements are in-situ meteorological masts with wind cup and vane anemometers on it [3]. But it is difficult to build on the sea due to high cost and complexity.
Because of the global growth of wind energy industry, more techniques, like ultrasonic anemometer, profilers and SODAR (Sonic Detection and Ranging) are increasingly being used to measure wind wake [4]. The measurement range based on relative air humidity, and the sensitivity is typically lower than lidar leading to much reduced data rates.

Comparing to the techniques above, the CDL has produced high spatial and temporal resolution measurements of wind fields [5]. The research of wind turbine wake detected by a continuous-wave CDL was done by Bingöl et al. [6] and Trujillo et al. [7]. CDL was put into turbine control study by QinetiQ to examine the requirements of a lidar system at Postlow in 2005[8].

Experimental investigations of the wind field in vicinity of wind turbine with the use of CDL were carried on in 2014. This paper describes the measurement process for the pulsed CDL that was performed to obtain wake parameters of the wind turbine wake, and presents some results of the field test under various atmospheric conditions.

\section{METHODOLOGY}

Lidar is one of the most accurate remote sensing techniques that transmit a laser beam into the atmosphere and the backscattered signal is detected. The1.5- $\mu \mathrm{m}$ all-fiber pulsed CDL with high resolution, which was utilized for the tests at mountain area, takes advantage of the fact that the frequency of the echo signal is shifted compared to the local-oscillator light because of the Doppler effect which occurs from backscattering of aerosols. The Doppler shift in the frequency of the backscattered signal is analyzed to obtain the line-of-sight (LOS) velocity component of the air motion. From the LOS velocities the characteristic of the turbulent wake can be deduced.

The OUC/ORSI CDL system is based on all-fiber laser technology and fast digital-signal-processing technology. Because of the all-fiber structure 
adoption, the system is stable, reliable and high-integrated. The spread of optical fiber communication caused drop in prices and mass production [9]. The Fast Fourier Transform (FFT) spectral estimates are real time processed with
FGPA. The 2-axis beam scanner and detection range of $3000 \mathrm{~m}$ enable the system to monitor the whole wind farming filed. The diagram of pulsed CDL setup is shown in Fig. 1. The main system specifications are summarized in Table 1 .

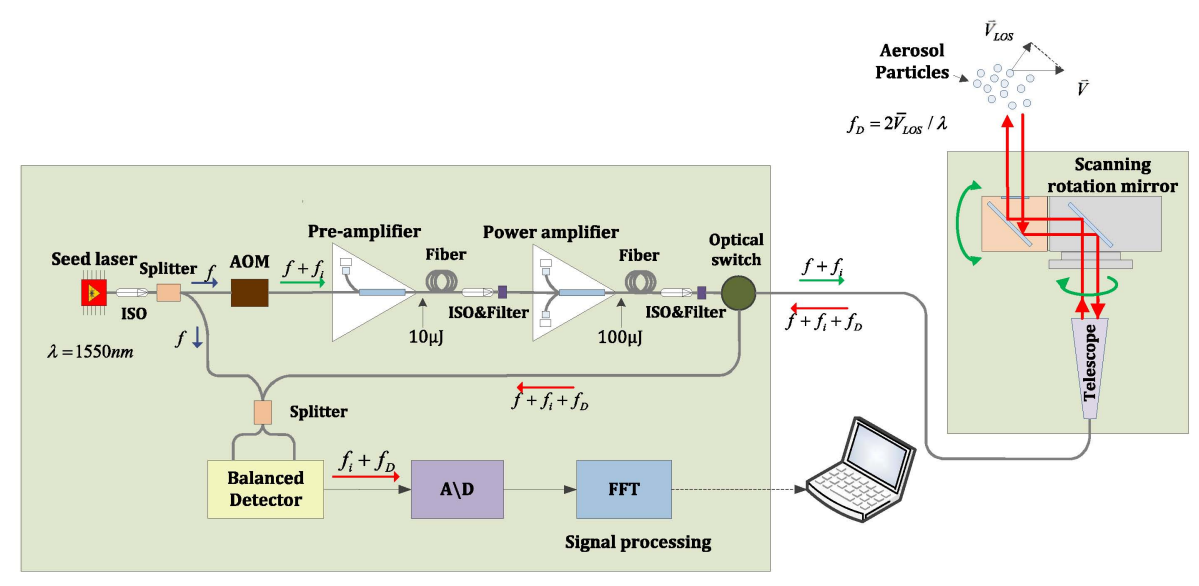

Fig. 1.The pulsed CDL setup. A 1.5- $\mu \mathrm{m}$ semi-conductor single frequency laser and an Erbium doped fiber amplifier is used as the transmitter. A fiber optical circulator and a telescope are used as the optical transceiver.

Table1.Specifications of the CDL.

\begin{tabular}{cc}
\hline CDL & Specification \\
\hline Wavelength & $1.5 \mu \mathrm{m}$ \\
Repetition rate & $10 \mathrm{kHz}$ \\
Pulse energy & $150 \mu \mathrm{J}$ \\
Speed measurement range & $\pm 50 \mathrm{~m} / \mathrm{s}$ \\
Speed accuracy & $\leq 0.3 \mathrm{~m} / \mathrm{s}$ \\
Scanner pointing accuracy & $0.1^{\circ}$ \\
Scan speed & $1-55^{\circ} / \mathrm{s}$ \\
Spatial resolution & $30 \mathrm{~m}$ \\
Measurement range & $30 \mathrm{~m} \mathrm{to} 3000 \mathrm{~m}$ \\
Power dissipation & $<150 \mathrm{~W}$ \\
weight & $<50 \mathrm{~kg}$ \\
\hline
\end{tabular}

\section{RESULTS}

The turbulent wake of wind turbine blades with different sizes from $50 \mathrm{~m}$ to $100 \mathrm{~m}$ had been observed by using the CDL in the detecting experiment. The lidar was located at the center of the area of interest. To measure the LOS component of the wind, two different scanning modes were applied: Plane-Position Indicator (PPI) scanning mode and Range-Height Indicator (RHI) scanning mode. For the PPI scanning, the elevation of the laser beam is constant and the azimuth angle is continuously changed. For the RHI scanning, the azimuth angle is not changed but the elevation is increased continuously.
In the field campaigns, the lidar was located from a sufficient distance from wind turbines so as to observe the upwind and leeward of the turbine. Taking into account the high accuracy of the spatialresolution of CDL, LOS velocity measurements of the wind turbines are shown below: one PPI scan (Fig.2) on January 3rd, 2014 and one RHI scan (Fig.3) on December 26th, 2013. The position of the wind turbines and the wind direction are indicated in the plot. The red and blue colors respectively indicate positive (away from lidar) and negative (towards lidar) movement of the particles along the laser beam. Fig. 2 shows a PPI scan through the rotor blades at mountain area. The width of lidar range gates is $60 \mathrm{~m}$. The scanning speed is $1 \% \mathrm{~s}$. The elevation is $2.00^{\circ}$ for this measurement. Because of the terrain effects, the turbulence caused by hills can be seen in Fig. 2. Low Signal Noise Ratio (SNR) makes the data unavailable near $3 \mathrm{~km}$. Hard target returns (when the laser pulse hits terrain) also decrease data availability. Fig. 3 shows a RHI scanning result. The scanning speed is $1 \%$ s. The elevation varied from $0^{\circ}$ to $20^{\circ}$ and the azimuth is $150^{\circ}$ for this measurement. 


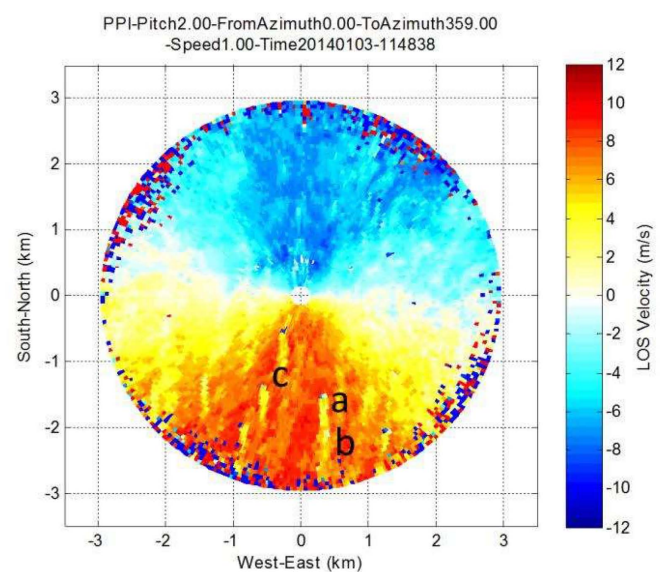

Fig.2. LOS wind field of wind farm by PPI scanning. The background wind is blowing from north to south. Wind direction and position of the different wind turbines are indicated in the figure. Turbine $b$ submerged under the turbulent wake of turbine a.

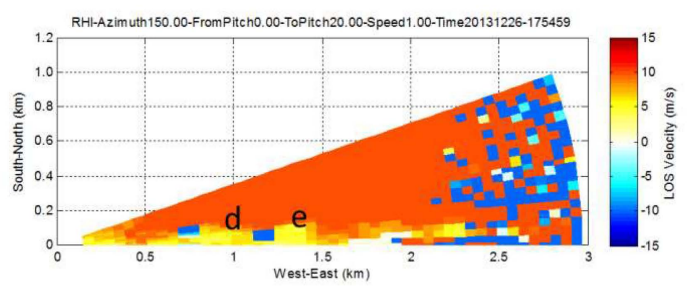

Fig.3. LOS velocity detected by RHI scanning mode through the rotor blades (two wind turbines). The wind is blowing from the northwest parallel to the laser beam. The position of the wind turbines $d$ and $e$ are at a range of $700 \mathrm{~m}$ and $1200 \mathrm{~m}$ respectively.

The rotor diameter is $80 \mathrm{~m}$ (1D) and hub height is $110 \mathrm{~m}$ in this case. The width of downwind turbulence is about $2 \mathrm{D}$ at the distance of $1 \mathrm{D}$ downwind of the rotor. In this observation, turbine wake lengths are from $3 \mathrm{D}$ to $10 \mathrm{D}$ depends on atmospheric conditions. The velocity deficit behind the wind turbine $\mathrm{c}$, e due to the wake vortices of turbine blades (derived from the PPI scanning mode in Fig. 2 and RHI scanning mode in Fig.3) are shown in Fig.4 and Fig.5, respectively. For wind turbine c, two rotor diameter range downwind of the LOS velocity at $50 \mathrm{~m}$ high is reduced about $54 \%$ in comparison to the maximum LOS velocity values. After 9D downwind is reduced about $14 \%$. For wind turbine e, one rotor diameter range downwind of the LOS velocity at height of $80 \mathrm{~m}$ is reduced about 52\%. After 5D downwind (10\% reduction of the LOS velocity at $80 \mathrm{~m}$ high), the wind field recovers slowly. For wind turbine $d$, because of the limited distance between two turbines, the maximum range of wake of turbine $d$ recorded in this measurement was only 3D. Find the vertical profile of LOS velocity in upwind and 1D range downwind respectively and calculate the velocity deficit. The velocity deficit at height of $40 \sim 80 \mathrm{~m}$ is about 55\% as shown in Fig. 6.

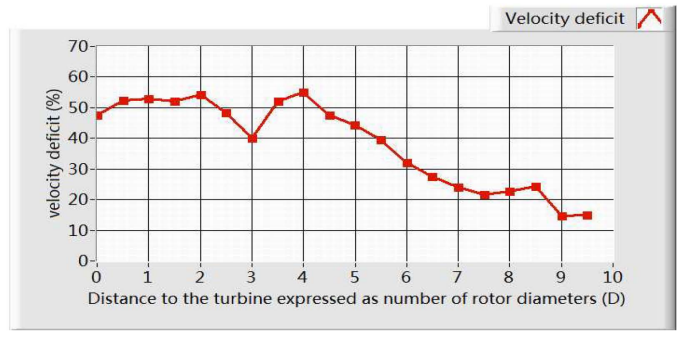

Fig.4.Velocity deficit downwind behind the wind turbine $\mathrm{c}$ as the function of the blade diameter $\mathrm{D}$ due to the wake vortices of turbine blade (derived from the PPI scanning mode in Fig.2).

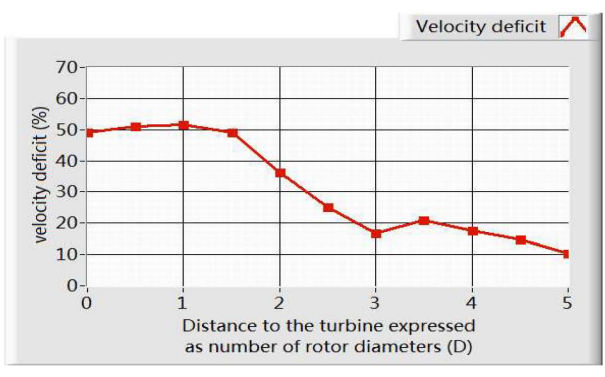

Fig.5.The velocity deficit downwind behind the wind turbine e due to the wake vortices of turbine blade (derived from the RHI scanning mode in Fig.3).

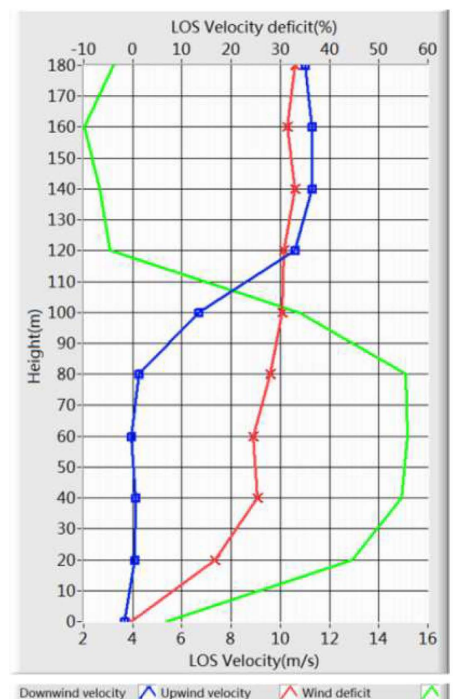

Fig.6.The vertical profile of velocity in upwind (red line) and downward (blue line) of turbine d. Green line is the vertical profile of velocity deficit up to $180 \mathrm{~m}$. 
The purpose for Yaw control of a turbine is to regulate the aerodynamic power. Here we focus on the turbulence wake dynamic when the yaw control happens. Fig.7 shows the change of turbulence wake before and after the yaw control. CDL can be used to measure velocity and take action to control wind turbine in advance.

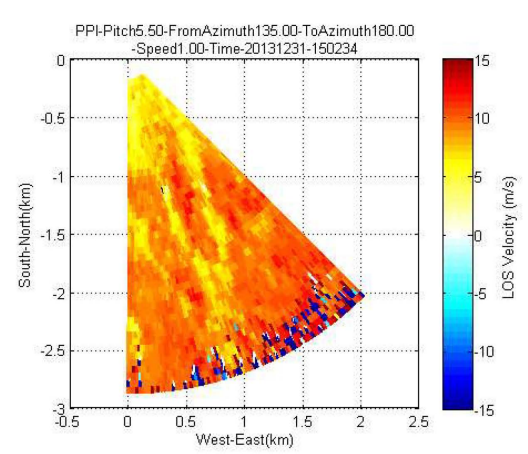

(a)

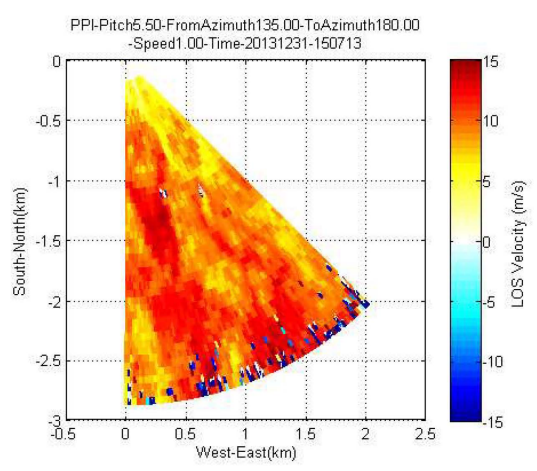

(b)

Fig. 7.The PPI scanning mode of LOS velocity shows that the scale of turbulence wake is much small after the yaw control. The width of lidar range gates is $30 \mathrm{~m}$. The scanning speed is $1 \%$ s. The elevation is $3.00^{\circ}$.

\section{CONCLUSIONS}

During the wind farming experiments in 2014, OUC/ORSI lidar team measured the wind speed and direction of a wind field and the wake parameters of wind turbines through LOS velocity obtained by the $1.5-\mu \mathrm{m}$ all-fiber pulsed CDL system. The paper shows that the $1.5-\mu \mathrm{m}$ CDL is a practical tool for wind energy research and a powerful technique for the wind energy industry. The subsequent significance is to improve the power output efficiency of the wind turbines and reduce dissipation. The CDL system can detect the interaction of wind turbines and will provide a new basis for wind farm site selection, design and optimization.

\section{ACKNOWLEDGEMENT}

This work was supported by NSFC under grant 41375016 and 41471309. The authors wish to thank Dr. Hongdong Zhu for valuable discussion on wind turbine technology. Thanks to the whole lidar group of Ocean University of China.

\section{REFERENCES}

[1]Frandsen, S., Barthelmie, R. J., Pryor, S., Rathmann, O., et al., 2006: Analytical modelling of wind speed deficit in large offshore wind farms, Wind Energy, 9, 39-53.

[2]Smalikho, I. N., et al., 2013: Lidar investigation of atmosphere effect on a wind turbine wake, J. Atmos. Oceanic Technol., 30, 2554-2570.

[3] Amar, F. B., Elamouri, M., Dhifaoui, R., 2008: Energy assessment of the first wind farm section of Sidi Daoud, Tunisia, Renewable Energy, 33, 2311-2321.

[4] Barthelmie, R., Folkerts, L., Larsen, G., et al., 2006: Comparison of wake model simulations with offshore wind turbine wake profiles measured by sodar, J. Atmos. Oceanic Technol., 23(7), 888-901.

[5]Högström, U., Asimakopoulos, D. N., Kambezidis, H., et al., 1987: A field study of the wake behind a $2 \mathrm{MW}$ wind turbine, Atmos. Environ., 22, 803-820.

[6]Bingöl, F., Mann, J., Larsen, G. C., 2010: Light detection and ranging measurements of wake dynamics, part I: one-dimensional scanning, Wind Energy, 13, 51-61.

[7] Trujillo, J.-J., Bingöl, F., Larsen, G. C., et al., 2011: Light detection and ranging measurements of wake dynamics. Part II: Two-dimensional scanning, Wind Energy, 14, 61-75.

[8]Jørgensen, H., Mikkelsen, T., Mann, J., Bryce, D., et al., 2004: Site wind field determination using a CW Doppler Lidar- comparison with cup anemometers at Risø, Proc. EWEA Special Topic Conference: The Science of Making Torque from Wind, The Netherlands, 261-266.

[9] Kameyama, S., Ando, T., Asaka, K., Hirano, Y., Wadaka, S., 2007: Compact all-fiber pulsed coherent Doppler lidar system for wind sensing, Appl. Opt. ,46(11), 1953-1962. 